\title{
FUZZY COGNITIVE MAP MODEL FOR SUPERVISORY MANUFACTURE SYSTEMS
}

\author{
C. D. Stylios and P. P. Groumpos \\ Laboratory for Automation and Robotics \\ Department of Electrical and Computer Engineering \\ University of Patras, Rion 26500, GREECE \\ Tel. +3061997293 Fax. +3061997309 \\ stylios@ee.upatras.gr
}

\begin{abstract}
A new approach to modeling the supervisor of manufacturing systems is proposed which it is based on Fuzzy Cognitive Map (FCM) Theory. In this paper the description and the construction of Fuzzy Cognitive Maps are examined. A two level control structure for manufacturing systems, where Supervisor is modeled as a FCM, is presented. Then, an FCM model for a process example is constructed and a supervisor consisted of two FCMs that are used for failure detection and decision analysis, is proposed. The application of soft computing methodologies such as FCM, in the field of manufacturing systems may contribute in the development of more autonomous and intelligent manufacturing systems.
\end{abstract}

Keywords

Supervisory Manufacturing Systems, Fuzzy Cognitive Maps, Soft Computing. 
During last decades Manufacturing Systems have utilized the advantages of computer and automation technology and they have made a great advance. The requirements of next generation manufacturing systems, which will be characterised by high autonomy and intelligence, have led engineers to investigate and invent new techniques that will integrate and combine known advanced methodologies and will be the core of these sophisticated systems. It has became quite clear that the requirements in the control and modeling systems cannot be met only with the existing conventional control theory. It is necessary to investigate and use new methods that will exploit past experience, will have learning capabilities, will be supplied with failure detection and identification characteristics and it will exploit imprecision and uncertainty. This is the case in large scale manufacturing systems and man-machine systems. One such new theory for modeling systems which is proposed in this paper and which will contribute to the effort for more intelligent control methods, is Fuzzy Cognitive Map (FCM) Theory. Fuzzy Cognitive Maps (FCM) is a new symbolic method for modelling supervisory manufacturing systems which relies on expert experience and follows the principle of "decreasing precision and increasing intelligence" (Saridis, 1989); therefore, this very promising methodology can be used to model and construct Intelligent Manufacturing Systems.

Fuzzy Cognitive Maps (FCM) can model dynamical complex systems that change with time following non-linear laws. They use a symbolic representation for the description and modeling of the system. FCM is consisted of concepts in order to illustrate different aspects in the behavior of the system and these concepts interact with each other showing the dynamics of the system. An FCM integrates the accumulated experience and knowledge on the operation of the system, as a result of the method by which it is constructed, i.e., using human experts that know the operation of system and its behavior.

Fuzzy Cognitive Maps have already been used to model behavioral systems in many different scientific areas. The introduction of cognitive maps made in political science (Axelrod, 1976) where they were used to represent social scientific knowledge and describe decision-making methods. Then, Kosko enhanced the power of cognitive maps considering fuzzy values for the concepts of cognitive map and fuzzy degrees of interrelationships between concepts (Kosko, 1986, 1992). After this pioneering work, Fuzzy Cognitive Maps attracted the attention of scientists in many fields and they have been used in a variety of different scientific problems. Fuzzy Cognitive Maps have been used for planning and making decisions in the field of international relations and political developments (Taber, 1991) and to model the behavior and reactions of virtual worlds (Dickerson, 1994). FCMs have been proposed as a generic system for decision analysis (Zhang, 1989) and as distributed cooperative agents (Zhang, 1992). In the control related themes, FCMs have been used to model and support a dynamic plant control (Goto, 1991), to represent Failure Models and Effects Analysis for a system model (Pelaez, 1996), 
and to model the supervisor of control systems (Stylios, 1998). It is obvious that there is high interest in the use of FCM in a wide range of different fields. In this paper the objective is to define and construct Fuzzy Cognitive Maps for modeling supervisory manufacturing systems, to represent a two-level hierarchical structure, to implement FCM in modeling a process and describe the structure of two FCMs that will be used to perform supervisory control with advanced characteristics.

\section{FUZZY COGNITIVE MAPS}

The synergistic and complementary use of fuzzy logic and neuro-computing has lend to the development of soft computing methodologies such as Fuzzy Cognitive Map (FCM). The graphical illustration of FCM is a signed directed graph with feedback, consisting of nodes and weighted interconnections. Nodes of the graph stand for the concepts that are used to describe the behavior of the system and they are connected by signed and weighted arcs representing the causal relationships that exist between the concepts (Figure 1). Each concept represents a characteristic of the system; in general it stands for states, variables, events, actions, goals, values, trends of the system which is modeled as an FCM. Each concept is characterized by a number $A_{i}$ that represents its value and it results from the transformation of the real value of the system's variable, for which this concept stands, in the interval $[0,1]$. It must be mentioned that all the values in the graph are fuzzy, and so weights of the interconnections belong to the interval $[-1,1]$. With the graphical representation of the behavioral model of the system, it becomes clear which state of the system (concept) influences other concepts and in which degree. This representation permits easily updating in the construction of the graph, such as the adding or deleting of an interconnection or a concept.

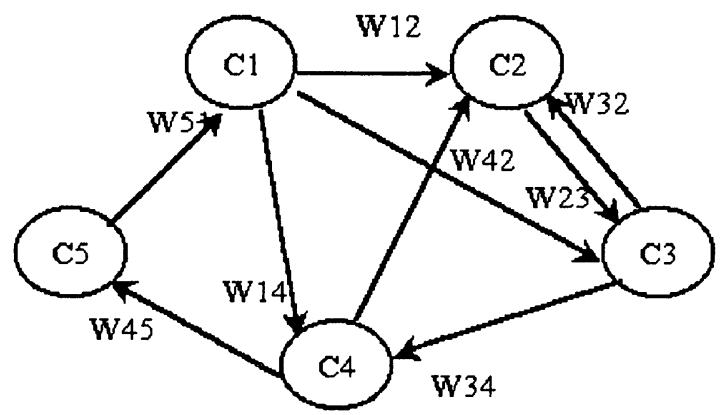

Figure 1. A simple Fuzzy Cognitive Map.

The knowledge of the dynamic behavior of the system is stored in the interconnections among concepts. The most important in describing the system is the determination of which concept influence other and with which degree. There 
are three possible types of causal relationships among concepts that express the type of influence from one concept to the others. The weight of the interconnection between concept $C_{i}$ and concept $C_{j}$, denoted by $W_{i j}$, could be positive for $\left(W_{i j} \succ 0\right)$ positive causality or there is negative causality $\left(W_{i j} \prec 0\right)$ or there is no relationship between concept $C_{i}$ and concept $C_{j}$, then $W_{i j}=0$.

The mathematical model, which describes an FCM, is very simple and it is consisted of vectors and matrices of dimension $n$ where $n$ equals the total number of distinct concepts of FCM. Thus there is a $1 \times n$ state vector $\mathbf{A}$, which includes the values of the $n$ concepts and a $n \times n$ weight matrix $\mathbf{W}$, which gathers the weights $W_{i j}$ of the interconnections among the $n$ concepts. The matrix $\mathbf{W}$ has $n$ rows and $n$ columns and the matrix diagonal is zero since it is assumed that no concept causes itself.

The value of each one concept is influenced by the values of the connected concepts with the appropriate weights and by its previous value. So the value $A_{i}$ for each concept $C_{i}$ is calculated by the following rule:

$$
A_{i}=f\left(\sum_{\substack{j=1 \\ j \neq i}}^{n} A_{j} W_{j i}+A_{i}^{\text {old }}\right)
$$

where $A_{i}$ is the value of concept $C_{i}$ at time $\mathrm{t}+1, A_{j}$ is the value of concept $C_{j}$ at time $\mathrm{t}, A_{i}^{\text {old }}$ is the value of concept $C_{i}$ at time $\mathrm{t}$, and $W_{j i}$ is the weight of the interconnection between $C_{j}$ and $C_{i}$, and $f$ is a threshold function. Threshold function squeezes the result of multiplication in the interval [0,1]. Equation (1) includes the old value of each concept, and so the FCM possesses memory capabilities and there is a smooth change after each recalculation.

\section{CONSTRUCTING FUZZY COGNITIVE MAPS}

The development and design of the appropriate Fuzzy Cognitive Map for the description of a system require the contribution of human knowledge. Experts and operators of the system know the behavior of the system and they have created in their mind a mental model of the system. This model can be easily transformed in a Fuzzy Cognitive Map. Experts are asked to determine the concepts that best describe the model of the system. Each concept of the FCM is a characteristic of the system, a state or a variable or input or an output of the system. Expert knows which factors are crucial for the modeling of the system and he represents a concept for each one. Moreover, during the operation of the system, he has observed which 
elements of the system influence others elements and for the corresponding concepts he determines the negative, positive or zero effect of one concept on the others. For each interconnection, it is assigned a fuzzy value, since it is assumed that there is a fuzzy degree of causation between concepts.

It is possible to have better results in the development of the FCM, if more than one expert is used. In that case, all experts are polled together and they determine the relevant factors and thus the concepts that should be presented in the map. Then, experts are individually asked to express the relationship among concepts, during the assigning of weights three parameters must be considered. Whether concept $C_{i}$ causes changes in concept $C_{j}$, or vice versa or there is no relationship between two concepts, how strongly concept $C_{i}$ influences concept $C_{j}$, which is the weight of the interconnection, and finally, what is the sign of the weight. Each expert will draw a Fuzzy Cognitive Map to describe the model and operation of the system, thus there will be a collection of individual FCMs that must be combined into a collective map.

If there is a large complex system, it could be necessary to construct more than one FCMs, each one describing different parts of the same system. And then, these different FCMs can be integrated and combined into one augmented FCM. In this way, connecting different Fuzzy Cognitive Maps can grow existing knowledge bases for different parts of the system.

It has just presented how a Fuzzy Cognitive Map is constructed; experts involved in the construction of FCM determine concepts and causality. In order to develop better FCM, learning algorithms, borrowed from the neural networks, can be utilized to adjust the weights of the interconnections among concepts and train the FCM. The weights of the causal edges change as if they were synapses in a neural network. A proposed algorithm is the Differential Hebbian learning method can be used (Kosko, 1992). The learning rule and the choosing of weights will determine the stability of FCM. The stability for FCM is proposed to be examined in terms of the eigenvalues of the weight connection matrix (Kosko, 1997). The stability of Fuzzy Cognitive Map needs further investigation, which is subject of future research work.

\section{TWO-LEVEL STRUCTURE FOR SUPERVISORY MANUFACTURING SYSTEMS}

The complex problems, that there are in Manufacturing Systems, require the development of intelligent systems that combine knowledge, techniques and methodologies from various sources. These intelligent systems are supposed to possess human-like expertise within a specific domain, adapt themselves and learn to do better in changing environments, and explain how they make decisions or take actions. 
Manufacturing Systems are complex systems for which it is impractical and impossible to construct an exact mathematical model describing by differential equations. For such systems, the human operator offers Supervisory Intelligent Control through the use of an imprecise and control methodology. The FCM is a symbolic representation for the description and modeling of complex systems, describing different aspects in the behavior of complex systems in terms of concepts; and interaction among concepts shows the dynamics of a system.

The proposed FCM methodology tries to establish the controller directly from experts who are controlling the process manually and successfully. The primary attention is paid to the human's behavior and experience, rather than to the process being controlled. This distinctive feature makes FCM applicable and attractive for dealing with the supervised problem where the process on the lower level is so complex that it is impossible or too expensive to derive a mathematical model; but the process is supervised and controlled satisfactory by human operators.

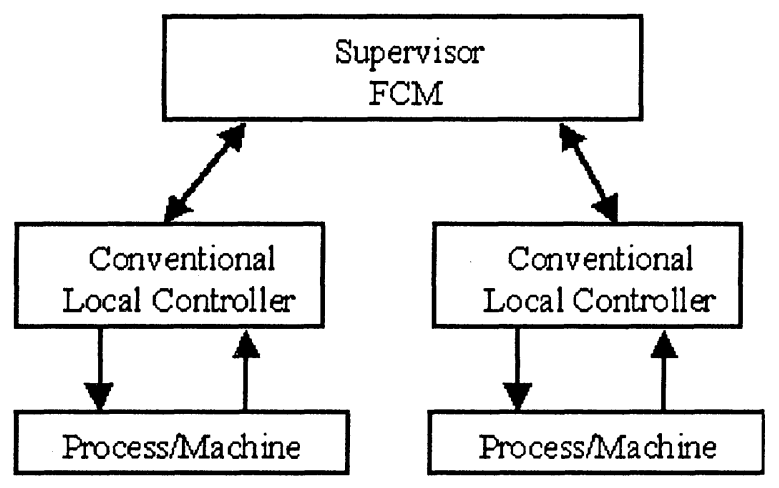

Figure 2. A two-level structure approach.

For the modeling of a manufacturing control system, a two level approach has been proposed in order to achieve a more sophisticated manufacturing system. Figure 2 depicts a two level structure that is used to model a general manufacturing system. Each machine/ process on the lower level has its own local controller that performs usual control actions. The supervisor is used for more generic purposes; to organize all the machines in order to accomplish a task, to help the operator make decisions, to planning strategically and to detect and analyze failures. Supervisor attempts to emulate such a human control capacity using an FCM (Stylios, 1998).

The role of the FCM is to extend the range of application of a conventional controller by using a more abstract representation of the process, general control knowledge and adaptation heuristics and to enhance the performance of the whole system. Thus, the FCM may replicate some of the knowledge and skills of the control engineer and it is built using a combination of knowledge representation techniques as causal models, production rules and object hierarchies. 


\section{FUZZY COGNITIVE MAP MODEL FOR MANUFACTURING SYSTEMS}

Knowledge on manufacturing plants includes the layout of the plant, the expected behavior of some parts of the plant, an aggregation of attributes or quality variables that are important. This information is captured using a Fuzzy Cognitive Map structure that exploits human operator's experience and knowledge. The expert knows the operation of the system and he has it stored in his memory in terms of concepts. He relates a process or a succession of processes to a concept, or a concept stand for a specific production procedure.

The most essential part is the drawing of an FCM, the determination of concepts that best describe the system, the direction and the grade of causality between concepts. So, the selection of the different factors of the system, that must be presented in the map, it must be the result of a close-up on system's operation behavior. Causality is another important part in the FCM design, it indicates whether a change in one variable causes change in another, and it must include the possible hidden causality that it could exist between several concepts. FCM model for a simple chemical process is constructed and illustrated in Figure 3. It is consisted of seven concepts and is developed by a human operator who supervises the process:

Concept 1: the state of Process 1;

Concept 2 : it represents the state of Pipeline which connects the two processes;

Concept 3 : the state of the Process2;

Concept 4: the Final Product of the two chemical processes;

Concept 5: the Quality of the Final Product;

Concept 6: the appearance of Failure 1, mostly related to Process 1;

Concept 7: the appearance of Failure 2, mostly related to Process 2.

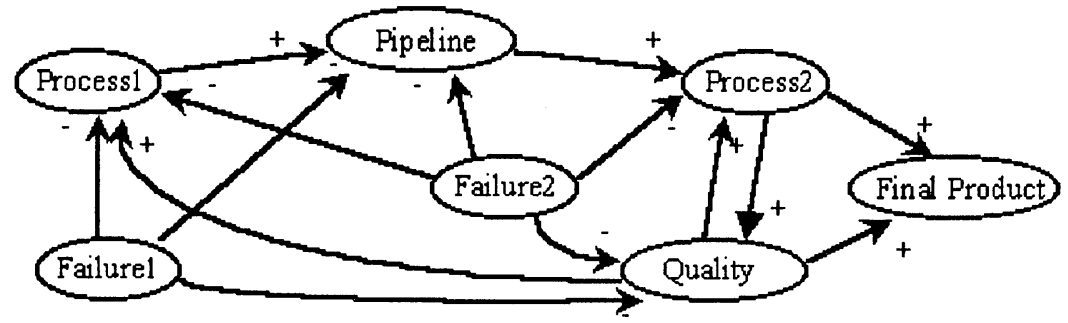

Figure 3. Fuzzy Cognitive Map of a simple chemical plant.

The operator of the system has associated the concepts according to their causal relationship. So Process 1 influences positively the concept of Pipeline. Pipeline influences positively the state of Process 2 and Process 2 influences positively the Final Product and the concept for the Quality of the final Product. The state of the Quality influences positively the Process 1 , Process 2 and Final Product. When 
Failure 1 appears, this influence negatively the Process 1 , and consequently the state of Pipeline which is depending on the Process 1 and the Quality of the Final product is influenced negatively. When, Failure 2 happens, this has negative effect on Process 2 and influence negatively the operation of Process 1 and Pipeline, as they are preprocessors of the Process 2. Failure 2 influences negatively the Quality of the final product.

After the initial drawing of the Fuzzy Cognitive Map, weights must be assigned to every causal relationship among concepts. Weights belong to the interval $[-1,1]$ or alternatively it could be used words weights like little or more or less, each concept take a fuzzy value in the interval $[0,1]$. When the FCM has been constructed, it is used to model the behavior and simulate the operation of the system. In each step of the simulation, values of concepts change according to the equation 1.

\section{STRUCTURE OF SUPERVISOR}

In complex industrial systems, it is very difficult to measure states and variables of the process that are good indicators of faults, and more elaborate and automatic measures are necessary. Usually operators of the system observe multiple data simultaneously and they make tough decisions based on their experience and empirical knowledge.

The proposed structure of supervisor is consisted of two Fuzzy Cognitive Maps (Figure 4). An FCM lies on the lower level and has just constructed; its concepts stand for complex and frequently observable faults, other represent measures, plain failures. In the figure, existing interactions among concepts are showing and are representing with fuzzy weights $W_{i j}$. Another one FCM is lying on the upper level and include concepts for decision making, planning and will give the appropriate commands to the process controller in the lower level. The decision making part of FCM evaluates alarm signals, process fail signals and other inputs and send control signals to the FCM on the lower level which influences the process.

The two FCMs will interact with each other and there will be an amount of information that must pass from the one FCM to the other. So, interface is consisted of two parts, one will pass information from the FCM in the lower level to the FCM in the upper level and the other interface in the opposite direction. This two-part interface is necessary because changes on two or more concepts in the FCM on the lower level could mean change in one concept on the upper level and the corresponding procedure, when information descends from the FCM on the upper level towards the lower level. Generally, information from two or more concepts of the FCM on the lower level pass through the interface and influence one concept in the FCM on the upper level, an analogous interface exists for the inverse transmission of information. 


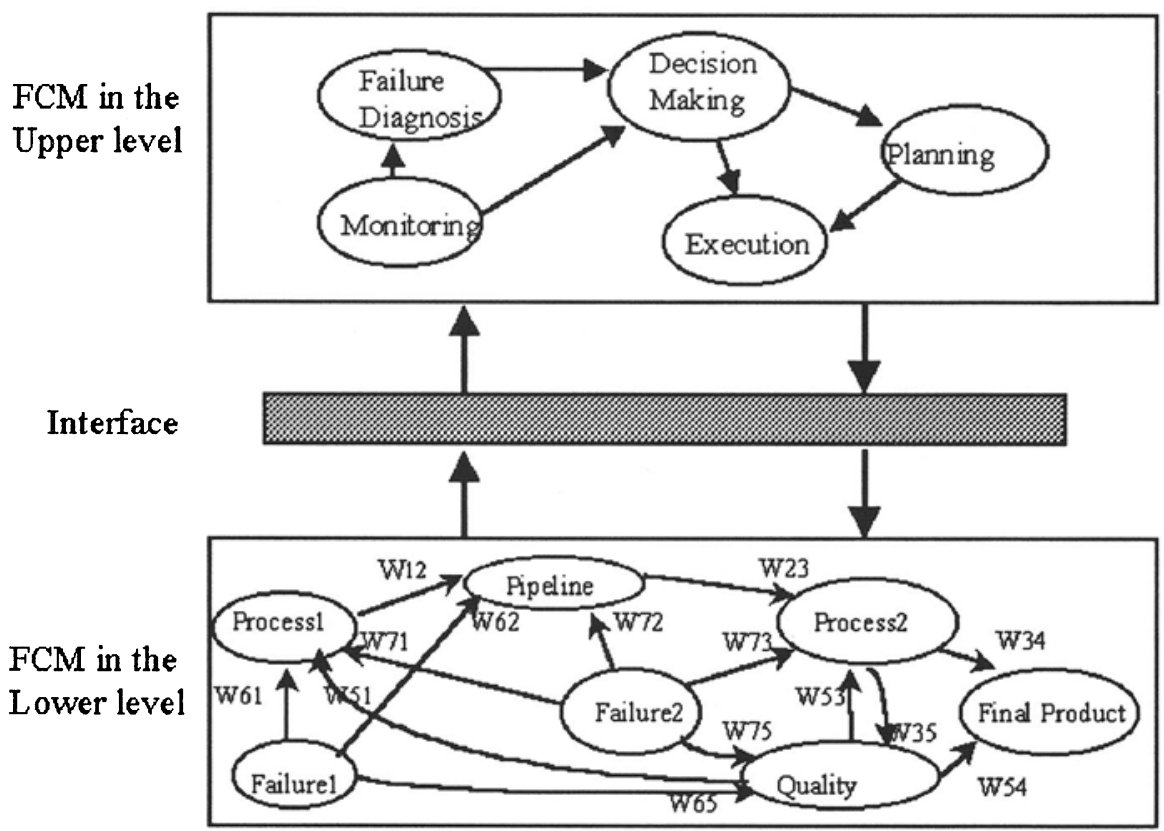

Figure 4. The two level structure controller with FCM on each level.

A more completed structure for the Fuzzy Cognitive Map that stand as the supervisor of the entire system and it is lying on the upper level, is subject of future research work. In this FCM, the structure of the decision making, planning and execution part will have been developed and the mechanism, with which the FCM in the lower level is influenced, will have been described.

\section{SUMMARY}

Fuzzy Cognitive Map, a new approach to modeling the behavior of complex manufacturing systems, which best utilizes existing experience and knowledge in the operation of the system, has been examined. For such systems it is extremely difficult to describe the entire system by a precise mathematical model. Thus, it is more attractive and useful to represent it, in a graphical way showing the causal relationships between states-concepts. Fuzzy Cognitive Maps appear to be an appealing tool in the description of the supervisor of complex systems, which can be complemented with other techniques and will lead to more sophisticated manufacturing systems. Future research may examine the application of FCM in real life systems and will investigate aspects such as the stability, controllability and the state space of model. 


\section{REFERENCES}

Axelrod, R. (1976) Structure of Decision: the Cognitive Maps of Political Elites. Princeton University Press, New Jersey.

Dickerson, J. A. and Kosko, B. (1994) Fuzzy virtual worlds as Fuzzy Cognitive Maps. Presence, 3, 173-189.

Goto, K. and Yamaguchi, T. (1991) Fuzzy associative memory application to a plant modeling. Proc. Intern Conf. on ANN, Espoo, Finland, 1245-8.

Kosko, B, (1986) Fuzzy Cognitive Maps. International Journal of Man-Machine Studies, 24, 65 - 75.

Kosko, B. (1992) Neural Networks and Fuzzy Systems. Prentice-Hall, New York.

Kosko, B. (1997) Fuzzy Engineering. Prentice-Hall, New Jersey.

Pelaez, C.E. and Bowles, J.B. (1996) Using Fuzzy Cognitive Maps as System Model for Failure Models, Effects Analysis. Information Sciences, 88, 177-199.

Saridis, G. (1989) Analytic formulation of the principle of Increasing Precision with Decreasing Intelligence for Intelligent Machines, Automatica, 25, 461-7.

Stylios, C. D. and Groumpos, P. P. (1998) The Challenge of modeling Supervisory Systems using Fuzzy Cognitive Maps. Journal Intelligent Manufacturing, 9.

Taber, R. (1991) Knowledge Processing with Fuzzy Cognitive Maps. Expert Systems with Applications, 2, 83-7.

Zhang, W.R., Chen, S. S. and Besdek, J.C. (1989) Pool2: a generic system for cognitive map development and decision analysis. IEEE Transactions on Systems, Man, and Cybernetics, 19, 31-9.

Zhang, W.R., Chen, S.S., Wang, W. and King, R. S. (1992) A Cognitive-MapBased approach to the coordination of distributed cooperative agents. IEEE Transactions on Systems, Man, and Cybernetics, 22, No1, 103-14.

\section{BIOGRAPHY}

Chrysostomos Stylios received the diploma in Electrical Engineering from the Aristotle University of Thessaloniki, Greece in 1992. He is currently studying for a Ph.D. degree on the subject of Hybrid Control of Hierarchical Systems, in the Dep. of Electrical \& Computer Engineering at the University of Patras. His interests include intelligent control, supervisory control. He is a member of IEEE and the National Technical Chamber of Greece.

Peter P. Groumpos received his Ph.D. in 1978 in Electrical Engineering from the State University of New York at Buffalo. He is a professor in the Dep. of Electrical \& Computer Engineering at the University of Patras and director of the Lab. for Automation \& Robotics. He was formerly on the faculty at Cleveland State University, USA 1979-1989. He was the director of the Communication Res. Lab. 1981-1986. Prof. Groumpos is the vice-president of the IFAC TC «Large Scale Systems». He is the Coordinator of the ESPRIT Network of Excellence ICIMSNOE. He has published over 70 papers. His main research interests are IMS, CIM, simulation, and hierarchical large-scale systems. 